\title{
Comparative survey on scolicidal effects of selenium and silver nanoparticles on protoscolices of hydatid cyst
}

\author{
Ahmad Nematollahi ${ }^{1, *}$, Parisa Shahbazi ${ }^{1}$, Abbas Rafat $^{2}$ and Morteza Ghanbarlu ${ }^{1}$ \\ ${ }^{l}$ Department of Pathobiology, Faculty of Veterinary Medicine, University of Tabriz, Iran \\ ${ }^{2}$ Department of Animal Science, Faculty of Agriculture, University of Tabriz, Iran
}

\begin{abstract}
Hydatidosis is a zoonotic diseases which affects human and livestock animals. The surgical treatment of hydatid diseases in man is still the most effective approach, but spillage of protoscolices is the risk of surgery. Therefore, it is essential to develop alternative approaches. Injection of protoscolicidal agents into the cysts preoperatively is an alternative method.The aim of the present study was the determination and comparison of lethal effects of silver nanoparticles (Ag-NPs) and selenium nanoparticles (Se-NPs) on protoscolices of hydatid cyst. Livers and lungs of sheep, which was naturally infected by hydatid cyst were collected from Tabriz slaughterhouse and transferred to the parasitology laboratory. Initially, the surface of cysts was disinfected by $70 \%$ Alcohol. Then, 25 ml of each cyst fluid were aspirated by sterile syringe and transferred to Erlenmeyer flask. After 30 minutes, the supernatant was discarded and protoscolices were washed with phosphate-buffered saline and tested by $1 \%$ eosin to assess their viability. Next, the samples of protoscolices, which were over $90 \%$, were selected and the effect of 50, 125, 250 and 500 ( $\mu \mathrm{g} / \mathrm{ml}$ ) of Se-NPs and Ag-NPs in the exposure times of 10, 20, 30 and 60 min were evaluated. Data were analysed by SAS software and analyzed by Duncan's comparison test. The significant differences were considered to be $p<0.0001$.The difference between the scolicidal effects of Se-NPs was statistically significant for all examined concentrations and exposure times compared to the control group $(p<0.0001)$. On the other hand, the scolicidal effect of Ag-NPs in 10 and 20 min exposer was not significant in comparison to the control group. Overall scolicidal effect of Se-NPs was higher than Ag-NPs and the difference was significant $(p<0.0001)$.The results of the present research indicated that it is possible to use Se-NPs as an effective scolicidal treatment. It is suggested the study of employing this compound in vivo or in combination with other procedures in treating hydatid cyst in further studies.
\end{abstract}

Keywords: Ag-NPs, Hydatid cyst, Nanoparticle, Se-NPs.

\begin{abstract}
Introduction
Echinococcosis, which is commonly called hydatidosis, is a zoonotic disease. This disease caused by larval stages (metacestodes) of the tapeworm Echinococcus granulosus and results in significant financial losses in livestock animals. Besides, it has led to a serious health problem in human in a number of countries (Eckert et al., 2001). Human cystic hydatidosis is a chronic and potentially life-threatening infection with the larval cystic stage (hydatid) of the dog taeniid tapeworm E. granulosus. Adult tapeworms can measure up to $2-7 \mathrm{~mm}$ long. Adult worms attach themselves to the walls of the small intestine of infected dogs and its eggs passed from feces of dogs and infested the environment. Following ingestion by a suitable intermediate host, e.g. human, sheep, and other livestock animals, the eggs hatch in the upper small intestine and hydatid cyst is formed in intermediate host's viscera, including the liver, and lungs, and less frequently in the muscle, kidney, bone, spleen, and other organs (Eckert and Deplazes, 2004). The surgical treatment of hydatid diseases (percutaneous aspiration,
\end{abstract}

injection, and respiration-PAIR) is still the most effective approach, which can be done successfully in a large number of patients if a cyst does not have a risky location. However the operation is risky when the cyst is either in multiple organs or in risky locations including in brain or other vital organs. In the other hand to reduce the risk of inoperative spillage protoscolices of cysts and formation of secondary cysts, alternative and low risky methods cab be replace surgery. Injection of protoscolicidal agents into the cysts preoperatively is an alternative method. These agents such as Ag-nitrate, cetrimide, hypertonic saline, and ethanol are employed to treat hydatid cyst but they have side effects such as toxemia, liver necrosis, and sclerosane colangititis (Rahimi et al., 2015). Therefore, it is essential to develop new scolicidal materials with more efficacies and low side effects, which can be implemented during surgery. It has currently been demonstrated that nanoparticles (NPs) have various unique properties due to their large surface-volume ratio. NPs are also able to enter cells more frequently than other particles. Among several metal NPs, which 
have been evaluated, one can name copper $(\mathrm{Cu})$, magnesium $(\mathrm{Mg})$, gold $(\mathrm{Au})$, zinc $(\mathrm{Zn})$, titanium $(\mathrm{Ti})$, and silver (Ag) (Retchkiman-Schabes et al., 2006; Khan et al., 2017). The synthesized silver NPs (Ag$\mathrm{Nps}$ ) were introduced as the most effective one with large capacity in direct contact with their environment and it has acceptable antimicrobial efficacy against parasites, viruses, and bacteria (Mahmoudvand et al., 2014). Few studies have shown that selenium NPs (SeNPs) can effectively inhibit the growth of some bacteria such as Staphylococcus aureus and pathogenic Escherichia coli (Yang et al., 2009; Tran and Webster, 2011). The aim of the study was to explore and compare the scolicidal effects of in vitro Ag-NPs and Se-NPs.

\section{Collection of protoscolices}

\section{Material and Methods}

Livers and lungs of sheep, which were infected naturally by hydatid cyst, were collected from Tabriz slaughterhouse, northwest of Iran. In the laboratory, the hydatid fluid was aspirated by a $20 \mathrm{ml}$ syringe and aseptically transferred into an Erlen Meyer flask. After $30 \mathrm{~min}$, the supernatant was discarded and the protoscolices were washed three times with phosphatebuffered saline ( $\mathrm{pH} 7.2)$ solution. The viability of the protoscolices was tested using eosin solution $(0.1 \%)$ under a light microscope. The concentration of protoscolices per $\mathrm{ml}$ of the hydatid fluid in normal saline solution $(0.9 \% \mathrm{NaCl})$ was detected. Samples containing $5000 \mathrm{ml}$ protoscolices with more than $90 \%$ viability were used.

\section{Characterization of the Se-NPs and Ag-NPs}

Se-NPs and Ag-NPs were purchased from Iranian Nanomaterials Pioneers Company, NANOSANY (Mashhad, Iran). The size of NPs was estimated to be 5-8 $\mathrm{nm}$ in diameter, metal basis and spherical. Deionized water was used to prepare desirable solutions.

\section{Scolicidal effects of the Se-NPs and Ag-NPs}

In the present study, different concentrations of $\mathrm{Ag}$ NPs and Se-NPs including 50, 125, 250, and 500 $(\mu \mathrm{g} / \mathrm{ml})$ were used for different exposure times including 10, 30 and 60 minutes. Initially, $0.5 \mathrm{ml}$ of the protoscolices solution was placed in test tubes. Then $0.5 \mathrm{ml}$ of various Se-NPs and Ag-NPs concentrations were added to each test tube. The tubes were incubated at $37^{\circ} \mathrm{C}$ for $10,20,30$, and 60 minutes. At the end of each incubation, the supernatant was removed. Next, 50 $\mu \mathrm{l}$ of $0.1 \%$ Eosin stain was added to each tube. After 5 minutes, protoscolices were smeared on a glass slide, were covered with a cover glass, and examined under a light microscope.

\section{Viability}

Stained protoscolices were considered as dead and unstained protoscolices were recorded as alive. The control group included non-treated protoscolices with the NPs.

\section{Statistical analysis}

Data were analysed by SAS software and means compared with Duncan's comparison test. The differences were considered significant at least $\mathrm{p}<0.05$.

\section{Results}

The scolicidal effect of various Se-NPs concentrations for different exposure times was shown in Fig 1. The difference between the scolicidal effects of Se-NPs was statistically significant for all examined concentrations and exposure times in comparison to the control group $(p<0.0001)$. Scolicidal effect of various Ag-NPs concentrations for a different exposed time was shown in Fig. 2. As seen in Figs. 1 and 2, the scolicidal effect of Ag-NPs in 10 and 20 min exposer was not significant in comparison to the control group. Overall scolicidal effect of Se-NPs was higher than Ag-NPs and these difference was significant $(p<0.0001)$ (Fig. 3).

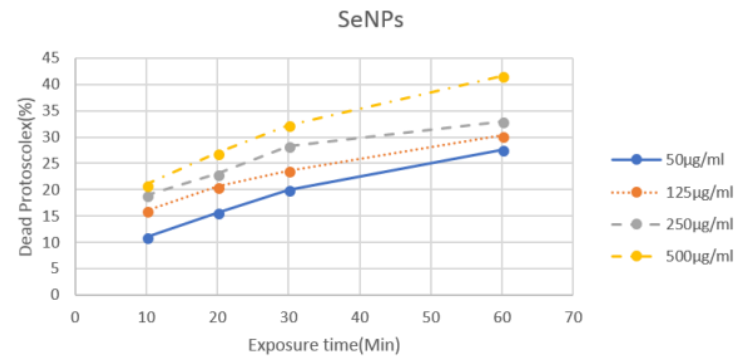

Fig. 1. Scolicidal effects of selenium nanoparticles (Se-NPs) against protoscolices of Echinococcus granulosus at the various concentrations following various exposure times.

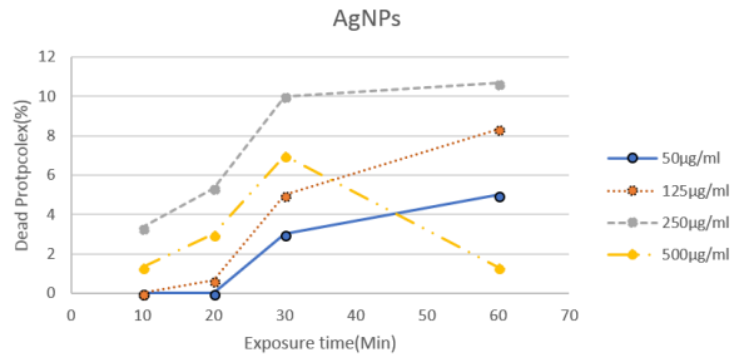

Fig. 2. Scolicidal effects of silver nanoparticles (Ag-NPs) against protoscolices of Echinococcus granulosus at the various concentrations following various exposure times.

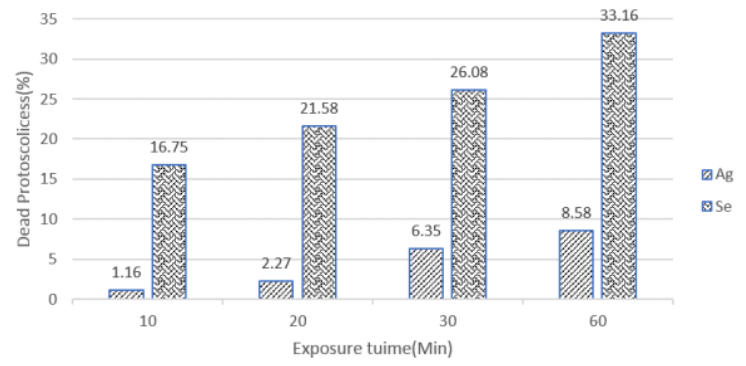

Fig. 3. Comparison on scolicidal effect of silver nanoparticles (Ag-NPs) and selenium nanoparticles (Se-NPs). 


\section{Discussion}

In this study, scolicidal effect of various dilutions $(50,125,250$, and $500 \mu \mathrm{g} / \mathrm{ml})$ of in vitro $\mathrm{Ag}$-NPs and Se-NPs in different exposure times $(10,20,30$, and $60 \mathrm{~min}$ ) were compared. Overall scolicidal effect of SeNPs was higher than Ag-NPs.

By considering the side effects of surgical approaches in the treatment of CHD, such as the formation of new cysts or death due to spillage of the cyst contents, injection, and respiration of scolicidal agents are suggested for replacing routines surgical methods (Yang et al., 2009). Many studies were performed on the scolicidal effect of chemical compounds such as hypertonic saline, silver nitrate, cetrimide, ethyl alcohol, $\mathrm{H}_{2} \mathrm{O}_{2}$, povidone iodine, Albendazole, and chlorohexidine gluconate. However, the majority of these compounds had side effects that limit their use in the treatment of in vivo hydatid cyst (Rouhani et al., 2013). Also in other studies, the scolicidal effect of plant extracts such as peganum harmala and Alium sativum (garlic), Zataria multiflora, Olea Europaea, Pistacia Atlantica and ephedra major was proved (Yetim et al., 2005; Nematollahi and Ghazi, 2014; Mahmoudvand et al., 2016; Zibaei et al., 2017). Recently many studies showed that NPs have potent scolicidal effect against protoscolices of hydatic cyst.

NPs are the wide class of materials that include particulate substances, which have one dimension less than $100 \mathrm{~nm}$ at least (Laurent et al., 2008). NPs have drawn increasing interest from every branch of medicine because of their ability to deliver drugs in the optimum dosage range, which often results in an increased therapeutic efficacy of the drugs, weakened side effects and improved patient compliance (Alexis $e t$ al., 2008).

The implication of NPs against parasites and other microorganism was shown in previous studies. Beheshti et al. (2013) reported the efficacy of biogenic Se-Nps against Leishmania major in vitro and in vivo. They showed that Se-NPs could inhibit the proliferation of the promastigote and amastigote forms of L.major and limit localized cutaneous leishmaniosis in an animal model (Beheshti et al., 2013). Mahmoudvand et al. (2014) used biogenic Se-NPs against protoscolices and proved potent scolicidal effects especially at concentrations of 500 and $250 \mathrm{mg} / \mathrm{mL}$. They showed that the scolicidal activity was $100 \%$ after 10 and 20 minutes of application, respectively (Mahmoudvand et al., 2014). Rahimi et al. (2015) used Ag-NPs against protoscolices and showed that the at all concentrations had high scolicidal effects. Besides, they emphasized that the concentrations of 0.1 and $0.15 \mathrm{mg} / \mathrm{mL}$ after 120 min of exposure times showed $83 \%$ and $90 \%$ mortality rate, respectively (Rahimi et al., 2015).

In the present study, both Se and Ag-NPs were used against protoscolices in vitro and the scolicidal effects of them were compared. The results of this study were proved that scolicidal effect of Se-NPs was higher than Ag-NPs. Besides, the results revealed that scolicidal effect at concentration $500 \mu \mathrm{g} / \mathrm{ml}$ was comparable with scolicidal effects of chemical compounds and plants extracts as previously described.

Although the silver compounds such as Ag-nitrate was reported as one of the most effective scolicidal agents, Lashkarzadeh et al. (2015) reported that Ag-NPs was less effective against protoscolices of hydatid cyst (Lashkarzadeh et al., 2015). A similar finding of AgNps was reported by other researchers. They exhibited that Ag-NPs were not as effective as Ag ions in killing Escherichia coli bacteria (Choi et al., 2008).

The results of present study showed a high effect of SeNPs on protoscolices of hydatid cyst. This result was similar to that of Beheshti et al. (2013), who proved that biogenic Se-NPs had toxic effect against Leishmania major in vitro (Beheshti et al., 2013). On the other hand, the safety of Se-NPs for living organisms was proved. For example, Shakibaie et al. (2013) showed this by oral administration of $10 \mathrm{mg} / \mathrm{kg}$ of Se-NPs to male mice (Shakibaie et al., 2013).

In conclusion, it is suggested the study of employing this compound in vivo or in combination with other procedures in treating hydatid cyst in further studies.

\section{Acknowledgment}

This work was financially supported by University of Tabriz.

\section{Conflict of interest}

The authors declare that there is no conflict of interest.

\section{References}

Alexis, F., Pridgen, E., Molnar, L.K. and Farokhzad, O.C. 2008. Factors affecting the clearance and biodistribution of polymeric nanoparticles. Mol. Pharm. 5, 505-515.

Beheshti, N., Soflaei, S., Shakibaie, M., Yazdi, M.H., Ghaffarian, F., Dalimi, A. and Shahverdi, A.R. 2013. Efficacy of biogenic selenium nanoparticles against Leishmania major: In vitro and in vivo studies. J. Trace Elem. Med. Biol. 27(3), 203-207.

Choi, O., Deng, K., Kim, N.J., Ross, L., Surampalli, R.Y. and Hu, Z. 2008. The inhibitory effects of silver nanoparticles, silver ions and silver chloride colloids on microbial growth. Water. Res. 42(12), 3066-3074.

Eckert, J., Deplazes, P., Craig, P.S., Gemmell, M.A., Gottstein, B., Heathn, D., Jenkind, D.J., Kamiya, M. and Lightowlers, M. 2001. Echinococcosis in animals: clinical aspects, diagnosis and treatment, in WHO/OIE Manual on echinococcosis in humans and animals: a public health problem of global concern, Eckert, J., Gemmell, M.A., Meslin, F., Pawłowski, Z.S., Editors. World Organization for Animal Health (OIE), Paris, pp: 89-90. 
Eckert, J. and Deplazes, P. 2004. Biological, epidemiological, and clinical aspects of echinococcosis, a zoonosis of increasing concern. Clin. Microbiol. Rev. 17, 107-135.

Khan, I., Saeed, K. and Khan, I. 2017. Nanoparticles: Properties, applications and toxicities. Arabian J. Chem. https://doi.org/10.1016/j.arabjc.2017.05.011

Lashkarzadeh, M.R., Asgharipour, K., Dezaki, E.S. and Fasihi-Harandi, M. 2015. Comparison of Scolicidal Effects of Amphotricin B, Silver Nanoparticles and Foeniculum vulgare Mill on Hydatid Cysts Protoscolices. Iran. J. Parasitol. 10, 206-212.

Laurent, S., Forge, D., Port, M., Roch, A., Robic, C., Vander, E. and Muller, R.N. 2008. Magnetic iron oxide nanoparticles: synthesis, stabilization, vectorization, physicochemical characterizations, and biological applications. Chem. Rev. 108(6), 2064-2110.

Mahmoudvand, H., Kheirandish, F., Ghasemi-Kia, M., Tavakoli, A. and Yarahmadi, M. 2016. Chemical composition, protoscolicidal effects and acute toxicity of Pistacia atlantica Desf. fruit extract. Nat. Prod. Res. 30, 1208-1211.

Mahmoudvand, H., Fasihi-Harandi, M., Shakibaie, M., Aflatooni, M.R., Ziaali, N., Makki, M.S. and Jahanbakhsh, S. 2014. Scolicidal effects of biogenic selenium nanoparticles against protoscolices of hydatid cysts. Int. J. Surg. 12, 399-403.

Nematollahi, A. and Ghazi, H. 2014. The in vitro study of comparison of scolicidal effectiveness of physical agents (temperature and smolarity), chemical agent (Albendazole) and aquatic extract of Harmel (Peganum Harmala L). Med. J. Tabriz Uni. 36, 76-83.

Rahimi, M.T., Ahmadpour, E., Rahimi-Esboei, B., Spotin, A., Kohansal, M.H., Alizadeh, A., Honary, S., Barabadi, H. and Mohahammadi, M. 2015.
Scolicidal activity of biosynthesized silver nanoparticles against Echinococcus granulosus protoscolices. Int. J. Surg. 19, 128-133.

Retchkiman-Schabes, P.S, Canizal, G., BecerraHerrera, R., Zorrilla, C., Liu, H.B. and Ascensio, J.A. 2006. Biosynthesis and characterization of Ti/Ni bimetallic nanoparticles. Opt. Mater. 29(1), 95-99.

Rouhani, S., Parvizi, P. and Spotin, A. 2013. Using specific synthetic peptide (p176) derived AgB 8/1$\mathrm{kDa}$ accompanied by modified patient's sera: a novel hypothesis to follow-up of cystic echinococcosis after surgery. Med. Hypotheses 81, 557-560.

Shakibaie, M., Shahverdi, A.R., Faramarzi, M.A., Hassanzadeh, G.R., Rahimi, H.R. and Sabzevari, O. 2013. Acute and subacute toxicity of novel biogenic selenium nanoparticles in mice. Pharm. Biol. 51(1), 58-63.

Tran, A.P. and Webster, T. 2011. Selenium nanoparticles inhibit Staphylococcus aureus growth. Int. J. Nanome. 6, 1553-1558.

Yang, J., Huang, K., Qin, S., Wu, X., Zhao, Z. and Chen, F. 2009. Antibacterial action of seleniumenriched probiotics against pathogenic Escherichia coli. Dig. Dis. Sci. 54, 246-254.

Yetim, I., Erzurumlu, K., Hokelek, M., Barıs, S., Dervisoglu, A., Polat, C., Belet, U., Buyukkarabacak, Y. and Guvenli, A. 2005. Results of alcohol and Albendazole injections in hepatic hydatidosis. J. Gastroenterol. Hepatol. 20(9), 14421447.

Zibaei, M., Salehi, S., Jafari, Z., Bahadory, S., Firoozeh, F. and Shahivand, M. 2017. In vitro assessment of the protoscolicidal activities of the Ephedra major methanol extracts. Int. J. Enteric Pathog. 5, 5-8. 